\title{
A study on the extreme value distribution of the minimum tensile strength of bolt
}

\author{
Geping $\mathrm{Bi}^{1, *}$, Jinzhe Chen ${ }^{2}$, and Wenhua Tai ${ }^{1}$ \\ ${ }^{1}$ China National Quality Supervision and Inspection Center for Knife, Scissors and Domestic Metal Tool, Yangjiang, Guangdong, \\ 529539, China \\ ${ }^{2}$ China National Accreditation Service for Conformity Assessment, Beijing, 100062, China
}

\begin{abstract}
Taking M12.5 $\times 1.25 \times 65-10.9$ bolt as an example, this paper studies the extreme distribution of the minimum value of bolt tensile strength in order to evaluate the reliability and stability of bolt product quality and to verify the production process. Through data collection, parameter estimation, distribution test and extreme prediction, it is concluded that: 1) the distribution of the minimum value of bolt tensile strength conforms to Gumbel extreme distribution; 2) when the return period is 10000 , the predicted minimum tensile strength is $1071.7 \mathrm{MPa} \pm 3.2 \mathrm{MPa}, k=2$. It is higher than the minimum value of $1040 \mathrm{MPa}$ required by ISO 898-1 standard.
\end{abstract}

\section{Introduction}

Fasteners are the most widely used standard parts in the mechanical industry. According to ISO 898-1:2013 standard [1], the tensile strength of fasteners cannot be lower than the specified value. When the chemical composition of the raw material is determined, the tensile strength of the fastener depends on the standardized production process, and the process evaluation is required before the standardized production process is determined.

The study of extreme value has a long history, the earliest research literature is 1824. At present, Gumbel maximum distribution has been widely used in finance, hydrology, architecture, bridge, metallurgy and other fields [2-9]. However, Gumbel minimum application research is less [2, 10-11].

The general requirements for nondestructive testing and metallographic structure defects of metal materials shall be less than or equal to the specified value, i.e. the maximum value shall not be exceeded, which belongs to the application research of maximum value distribution. The mechanical properties of metal materials, such as strength, plasticity, hardness, impact absorbed energy, fatigue and so on, are generally required to be greater than or equal to the specified value, i.e. not less than the minimum value, which belongs to the application research of minimum value distribution. The application research of minimum value has important guiding significance in process design, process evaluation, process control, product quality evaluation and so on.

In this paper, samples were randomly collected from M12.5 $\times 1.25 \times 65-10.9$ bolts made of $35 \mathrm{CrMo}$ by a specific process. The distribution of the minimum tensile strength of the bolts was studied. Predict the minimum tensile strength that may occur for the production department to evaluate the reliability and stability of bolt product quality and to verify the specific production process.

\section{Data collection}

Twenty four groups of testing data of M12.5 $\times 1.25 \times$ 65-10.9 bolts were collected from the testing history samples. Five tensile strength bolts were tested in each group. The testing was conducted according to Clause 9.2 of ISO 898-1:2013 standard [1]. See Table 1 for the data of tensile strength samples.

The minimum values of 24 groups of tensile strength in Table 1 are arranged in non descending order, which are $x_{(1)} \sim x_{(24)}$, and $x_{(1)} \leq x_{(2)} \leq \ldots \leq x_{(24)}$, see the "Data collection" column of Table 2 .

The sample average value $(A v g)$ of the minimum value is $1130.3 \mathrm{MPa}$, the sample standard deviation $(S)$ is $8.42 \mathrm{MPa}$, the sample skewness coefficient $\left(b_{\mathrm{s}}\right)$ is $-0.4 \leq 0$, and the sample kurtosis coefficient $\left(b_{\mathrm{k}}\right)$ is $2.0 \leq 3$. See the last row of Table 2. From the skewness coefficient and kurtosis coefficient of the sample, we can see that: 1) the distribution of the minimum tensile strength at the low value range tends to deviate from the center more than that at the high value range; 2) it may have a few small extreme values; 3 ) the minimum value is not concentrated near the average value; 4) it has a long left tail [11-12].

For the minimum value distribution in Gumbel extreme value distribution, when $x \rightarrow-\infty$, the density function of the minimum value distribution decreases exponentially [2]. In this paper, it is assumed that the minimum value distribution of bolt is consistent with

\footnotetext{
${ }^{*}$ Corresponding author: bigeping@sina.com
} 
Gumbel extreme value distribution firstly.

Table 1. Sample data of bolt tensile strength / MPa

\begin{tabular}{c|c|c|c|c|c|c|c|c|c|c|c|c}
\hline № & $\mathbf{0 1}$ & $\mathbf{0 2}$ & $\mathbf{0 3}$ & $\mathbf{0 4}$ & $\mathbf{0 5}$ & $\mathbf{0 6}$ & $\mathbf{0 7}$ & $\mathbf{0 8}$ & $\mathbf{0 9}$ & $\mathbf{1 0}$ & $\mathbf{1 1}$ & $\mathbf{1 2}$ \\
\hline 1 & 1152 & 1154 & 1140 & 1171 & 1157 & 1141 & 1143 & 1146 & 1139 & 1134 & 1144 & 1140 \\
\hline 2 & 1151 & 1145 & 1149 & 1130 & 1121 & 1141 & 1133 & 1152 & 1152 & 1145 & 1147 & 1144 \\
\hline 3 & 1139 & 1130 & 1136 & 1141 & 1151 & 1140 & 1147 & 1154 & 1136 & 1126 & 1138 & 1148 \\
\hline 4 & 1134 & 1159 & 1137 & 1155 & 1130 & 1149 & 1130 & 1144 & 1129 & 1150 & 1139 & 1141 \\
\hline 5 & 1142 & 1129 & 1138 & 1161 & 1133 & 1139 & 1145 & 1138 & 1159 & 1134 & 1151 & 1163 \\
\hline Min & 1134 & 1129 & 1136 & 1130 & 1121 & 1139 & 1130 & 1138 & 1129 & 1126 & 1138 & 1140 \\
\hline № & $\mathbf{1 3}$ & $\mathbf{1 4}$ & $\mathbf{1 5}$ & $\mathbf{1 6}$ & $\mathbf{1 7}$ & $\mathbf{1 8}$ & $\mathbf{1 9}$ & $\mathbf{2 0}$ & $\mathbf{2 1}$ & $\mathbf{2 2}$ & $\mathbf{2 3}$ & $\mathbf{2 4}$ \\
\hline 1 & 1141 & 1151 & 1160 & 1143 & 1140 & 1139 & 1143 & 1155 & 1132 & 1153 & 1112 & 1160 \\
\hline 2 & 1138 & 1136 & 1140 & 1145 & 1138 & 1159 & 1131 & 1154 & 1137 & 1153 & 1126 & 1150 \\
\hline 3 & 1142 & 1141 & 1153 & 1146 & 1147 & 1165 & 1137 & 1162 & 1155 & 1144 & 1125 & 1152 \\
\hline 4 & 1121 & 1165 & 1151 & 1154 & 1148 & 1136 & 1156 & 1124 & 1123 & 1141 & 1132 & 1156 \\
\hline 5 & 1122 & 1143 & 1118 & 1143 & 1135 & 1144 & 1154 & 1171 & 1120 & 1152 & 1143 & 1120 \\
\hline Min & 1121 & 1136 & 1118 & 1143 & 1135 & 1136 & 1131 & 1124 & 1120 & 1141 & 1112 & 1120 \\
\hline
\end{tabular}

\section{Parameter estimation}

The distribution function and probability density function of Gumbel minimum distribution are formula (1) and formula (2), respectively. The empirical guarantee function of Gumbel is formula (3), and the natural logarithm maximum likelihood function of Gumbel distribution samples is formula (4).

$$
\begin{aligned}
& F(x)=1-e^{-e^{\frac{(x-\lambda)}{\sigma}}} \\
& f(x)=\frac{1}{\sigma} e^{\frac{(x-\lambda)}{\sigma}-e^{\frac{(x-\lambda)}{\sigma}}} \\
& F\left(x_{i}\right)=P\left\{X \leq x_{i}\right\}=p_{i}=\frac{i}{n+1} \quad i=1, \cdots, n \\
& L L(x, \lambda, \sigma)=\sum_{i=1}^{N}\left[\operatorname{Ln}\left(\frac{1}{\sigma}\right)+\frac{(x-\lambda)}{\sigma}-e^{\frac{(x-\lambda)}{\sigma}}\right]
\end{aligned}
$$

In formula (1), (2), (3) and (4), where $\infty<x<\infty, \infty<\lambda$ $<\infty, \sigma$ is the scale parameter, $\lambda$ is the location parameter, $X$ is the random variable, $x$ is the value of $X, n$ is the number of samples used for the evaluation of extreme distribution parameters, $p_{\mathrm{i}}$ is the empirical cumulative density [2, 11-13].

Set:

$y=\frac{x-\lambda}{\sigma}$

Then:

$x=\sigma y+\lambda$

Formula (1), (2) and (5) can be used to deduce:

$$
\begin{aligned}
& F(x)=1-e^{-e^{y}} \\
& f(x)=\frac{1}{\sigma} e^{y-e^{y}}
\end{aligned}
$$

Formula (6) and (7) can further deduce:

$$
\begin{aligned}
& y=\operatorname{Ln}\{-\operatorname{Ln}[1-F(x)]\} \\
& x=\sigma y+\lambda=\sigma \operatorname{Ln}\{-\operatorname{Ln}[1-F(x)]\}+\lambda \\
& x_{p}=\sigma y+\lambda=\sigma \operatorname{Ln}[-\operatorname{Ln}(1-p)]+\lambda
\end{aligned}
$$

In formula (11), $x_{p}$ is called the $p$-quantile of distribution function $F(x) . p$ is the probability associated with the $p$-quantile of $F(x)$ [12].

If $T$ is the return period, the minimum strength of the bolt predicted at the return period $T$ is:

$x(T)=\sigma \operatorname{Ln}\left[-\operatorname{Ln}\left(1-\frac{1}{T}\right)\right]+\lambda$

In formula (10-12), there are two unknown parameters, scale parameter $\sigma$ and location parameter $\lambda$. Two unknown parameters were evaluated by the maximum likelihood method [2-11]. The specific methods are as follows:

1) The minimum value samples of bolts in Table 1 are arranged in non descending order.

2) The empirical cumulative density $p_{\text {i }}$ of each sample is calculated by formula (3).

3) $y_{\mathrm{i}}$ is calculated from formulas (3) and (9).

4) Set the average value as the initial value of $\lambda$, and the standard deviation as the initial value of $\sigma$, that is, $\lambda=$ 1130.3 MPa, $\sigma=8.42 \mathrm{MPa}$. From formula (4), the component $L\left(x_{\mathrm{i}}\right)$ of the maximum likelihood function of the natural logarithm of the sample is calculated in turn.

5) The maximum likelihood function $\operatorname{LL}(x, \lambda, \sigma)$ is calculated from formula (4).

6) Using the Excel's "planning solving" function, the $\sigma$ and $\lambda$ that make $L L$ get the maximum value are calculated by Newton iterative method. From this, It can be concluded that: $L L\left(x, \lambda_{M L}, \sigma_{M L}\right)=-83.863, \sigma_{M L}=6.79$, $\lambda_{M L}=1134.2 . \sigma_{M L}$ and $\lambda_{M L}$ are scale and location parameters calculated by maximum likelihood method. 
The above keyabbreviations, symbols and datas are in the "parameter estimation" column and the last row in Table 2.

Through the parameter evaluation, the extremum distribution function and probability density function of the minimum value of bolt tensile strength are formula (13) and formula (14), respectively.

$$
F(x)=1-e^{-\frac{(x-1134,2)}{6.79}}
$$

$$
f(x)=\frac{1}{6.79} e^{\frac{(x-1134.2)}{6.79}-e^{\frac{(x-1134.2)}{6.79}}}
$$

\section{Distribution test}

\begin{tabular}{|c|c|c|c|c|c|c|c|c|c|}
\hline \multicolumn{3}{|c|}{ Data collection } & \multicolumn{3}{|c|}{ Parameter estimation } & \multicolumn{3}{|c|}{$\mathrm{K}-\mathrm{S}$ test } & \multirow{2}{*}{$x_{\mathrm{i}}(T)$} \\
\hline $\mathrm{i}$ & $x_{\mathrm{i}}$ & $\mathrm{Min} / \mathrm{MPa}$ & $p_{\mathrm{i}}$ & $y_{\mathrm{i}}$ & $L\left(x_{\mathrm{i}}\right)$ & $F \mathrm{n}\left(x_{\mathrm{i}}\right)$ & $F_{\mathrm{O}}\left(x_{\mathrm{i}}\right)$ & $D n(i)$ & \\
\hline 1 & $x_{1}$ & 1112 & 0.04 & -3.1985 & -5.226 & 0.000 & 0.037 & 0.0373 & 1112.5 \\
\hline 2 & $x_{2}$ & 1118 & 0.08 & -2.4843 & -4.396 & 0.042 & 0.088 & 0.0462 & 1117.3 \\
\hline 3 & $\mathrm{x}_{3}$ & 1120 & 0.12 & -2.0570 & -4.133 & 0.083 & 0.116 & 0.0329 & 1120.2 \\
\hline 4 & $x_{4}$ & 1120 & 0.16 & -1.7467 & -4.133 & 0.125 & 0.116 & 0.0505 & 1122.3 \\
\hline 5 & $x_{5}$ & 1121 & 0.20 & -1.4999 & -4.005 & 0.167 & 0.133 & 0.0750 & 1124.0 \\
\hline 6 & $x_{6}$ & 1121 & 0.24 & -1.2930 & -4.005 & 0.208 & 0.133 & 0.1166 & 1125.4 \\
\hline 7 & $x_{7}$ & 1124 & 0.28 & -1.1132 & -3.643 & 0.250 & 0.200 & 0.0921 & 1126.6 \\
\hline 8 & $x_{8}$ & 1126 & 0.32 & -0.9528 & -3.424 & 0.292 & 0.258 & 0.0750 & 1127.7 \\
\hline 9 & $x_{9}$ & 1129 & 0.36 & -0.8068 & -3.148 & 0.333 & 0.372 & 0.0385 & 1128.7 \\
\hline 10 & $x_{10}$ & 1129 & 0.40 & -0.6717 & -3.148 & 0.375 & 0.372 & 0.0448 & 1129.6 \\
\hline 11 & $x_{11}$ & 1130 & 0.44 & -0.5450 & -3.074 & 0.417 & 0.417 & 0.0418 & 1130.5 \\
\hline 12 & $x_{12}$ & 1130 & 0.48 & -0.4248 & -3.074 & 0.458 & 0.417 & 0.0835 & 1131.3 \\
\hline 13 & $x_{13}$ & 1131 & 0.52 & -0.3093 & -3.012 & 0.500 & 0.464 & 0.0774 & 1132.1 \\
\hline 14 & $x_{14}$ & 1134 & 0.56 & -0.1973 & -2.916 & 0.542 & 0.621 & 0.0796 & 1132.9 \\
\hline 15 & $x_{15}$ & 1135 & 0.60 & -0.0874 & -2.922 & 0.583 & 0.675 & 0.0920 & 1133.6 \\
\hline 16 & $x_{16}$ & 1136 & 0.64 & 0.0214 & -2.953 & 0.625 & 0.728 & 0.1034 & 1134.3 \\
\hline 17 & $x_{17}$ & 1136 & 0.68 & 0.1305 & -2.953 & 0.667 & 0.728 & 0.0618 & 1135.1 \\
\hline 18 & $x_{18}$ & 1136 & 0.72 & 0.2413 & -2.953 & 0.708 & 0.728 & 0.0216 & 1135.8 \\
\hline 19 & $x_{19}$ & 1138 & 0.76 & 0.3557 & -3.104 & 0.750 & 0.826 & 0.0762 & 1136.6 \\
\hline 20 & $x_{20}$ & 1138 & 0.80 & 0.4759 & -3.104 & 0.792 & 0.826 & 0.0346 & 1137.4 \\
\hline 21 & $x_{21}$ & 1139 & 0.84 & 0.6057 & -3.233 & 0.833 & 0.868 & 0.0350 & 1138.3 \\
\hline 22 & $x_{22}$ & 1140 & 0.88 & 0.7515 & -3.407 & 0.875 & 0.905 & 0.0296 & 1139.3 \\
\hline 23 & $x_{23}$ & 1141 & 0.92 & 0.9265 & -3.631 & 0.917 & 0.934 & 0.0241 & 1140.5 \\
\hline 24 & $x_{24}$ & 1143 & 0.96 & 1.1690 & -4.266 & 0.958 & 0.974 & 0.0259 & 1142.1 \\
\hline
\end{tabular}

Table 2. Sample analysis and calculation of minimum tensile strength of bolt

Whether the theoretical distribution function and the theoretical density function of the minimum tensile strength of bolts are consistent with the actual needs distribution test. In this paper, K-S test method [2-9] is used. See Table 2 for the process data of K-S test. The specific methods are as follows:

1) Calculate the empirical distribution function $F \mathrm{n}\left(x_{\mathrm{i}}\right)=(i-1) / \mathrm{n}$;

2) Calculate the theoretical distribution function $F_{\mathrm{O}}\left(x_{\mathrm{i}}\right)$ according to formula (13).

3) Calculation statistics $D \mathrm{n}(\mathrm{i}), D \mathrm{n}(\mathrm{i})=\max \left(\mid F \mathrm{n}\left(x_{\mathrm{i}}\right)\right.$ $\left.F_{\mathrm{O}}\left(x_{\mathrm{i}}\right)||, i / \mathrm{n}-F_{\mathrm{O}}\left(x_{\mathrm{i}}\right) \mid\right)$.

4) Let $D n$ be the maximum of $D n(i)$, with $D n=$ $\operatorname{Dn}(16)=0.1034$.
5) In the case of $n=24$ and significance level of $95 \%$, the critical value of K-S test $D_{24,0.05}=0.2776$ can be obtained.

It can be seen that $D n=0.1166<D_{24,0.05}=0.2776$, so it can be determined that the theoretical distribution function and theoretical density function formula (13-14) of the minimum value of the bolt tensile strength are consistent with the actual extreme value distribution.

The above key abbreviations, symbols and datas are in the " K-S test " column and the last row in Table 2.

The distribution curve of the minimum value of the bolt tensile strength obtained according to formula (13-14) is shown in Figure 1. 


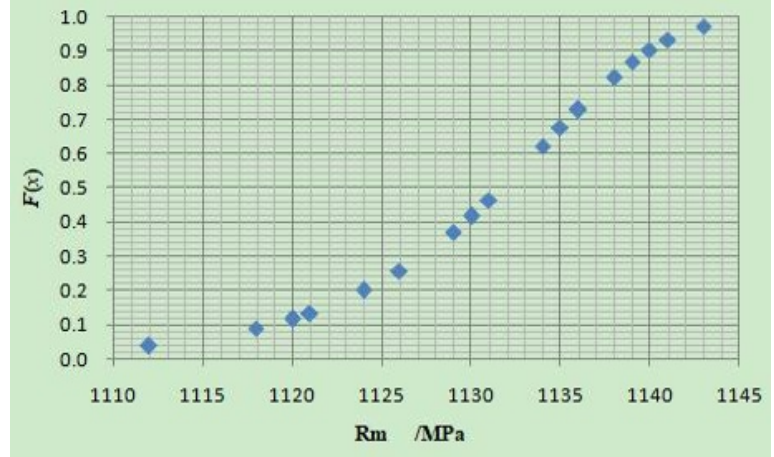

a)

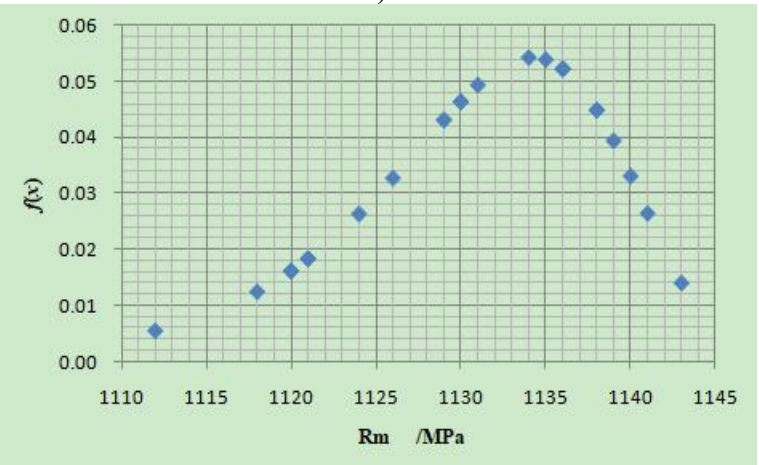

b)

Fig.1. Distribution of minimum tensile strength of bolt. a) Distribution curve of minimum; b) Probability density curve of minimum

\section{Mimimum value prediction}

According to the theoretical extreme value distribution function formula (13-14) and formula (12) of minimum value of bolt tensile strength, it can be concluded that the prediction function of minimum value of bolt strength with return period of $T$ is formula (14):

$$
\begin{aligned}
& x(T)=6.79 \operatorname{Ln}\left[-\operatorname{Ln}\left(1-\frac{1}{T}\right)\right]+1134.2 \\
& S E=\sqrt{\frac{\sum_{i=1}^{n}\left[x_{i}-x_{i}(T)\right]^{2}}{n-1}}
\end{aligned}
$$

According to formula (14), the theoretical value corresponding to 24 samples can be calculated, as shown in $x_{\mathrm{i}}(T)$ in Table 2 . The fitting standard deviation between the theoretical value and the actual value of the sample is calculated according to formula (15) [14], then the expanded uncertainty $(U)$ of the theoretical value is $\pm k \times S E$. After calculation, $S E=1.6 \mathrm{Mpa}, 95 \%$ confidence interval: $U= \pm 3.2 \mathrm{MPa}, \quad k=2$.

Figure 2 is the quantile plot of the actual value and theoretical value of the sample, i.e. Q-Q Plot. The dotted line in the figure is the upper and lower limit of the expanded uncertainty.

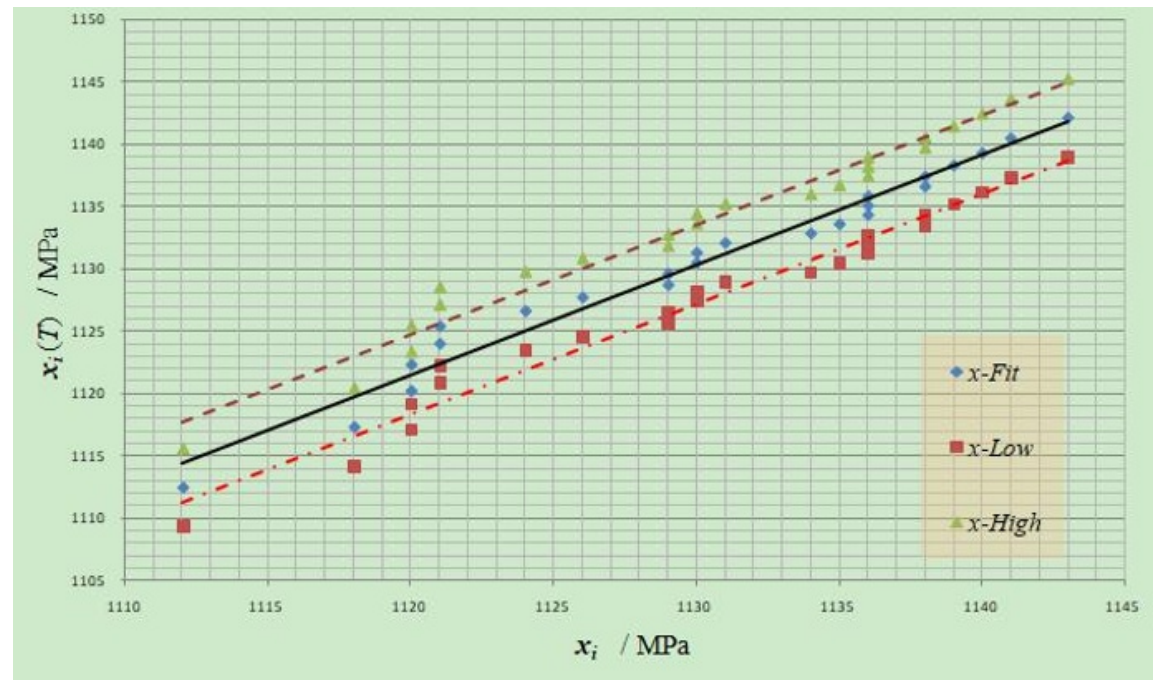

Fig.2. Q-Q Plot

Through the calculation and analysis of the extreme value of 24 groups of samples, it can be predicted that when the return period $T=10000$, the minimum value of bolt tensile strength $(\mathrm{Rm})$ is $1071.7 \mathrm{MPa} \pm 3.2 \mathrm{MPa}, k=2$. That is to say, for M12.5 $\times 1.25 \times 65-10.9$ bolt made of $35 \mathrm{CrMo}$, the technical requirement [1] is $\mathrm{Rm} \geq 1040 \mathrm{MPa}$. When the return period is 10000 , the minimum $\mathrm{Rm}$ is higher than the technical requirement. It can be determined that the quality of the bolt is stable and reliable and the specific production process is reliable. The prediction results can be explained as follows: For every 10000 samples, the probability of $\mathrm{Rm} \leq 1071.7$ $\mathrm{MPa} \pm 3.2 \mathrm{MPa}$ is $1 / 10000$, i.e. $0.01 \%$. Or, $\mathrm{Rm} \leq 1071.7$
$\mathrm{MPa}$ 土3.2 MPa will appear in every 10000 samples; Or, in every 10000 samples, $\mathrm{Rm}$ of each sample will be less than $1071.7 \mathrm{MPa} \pm 3.2 \mathrm{MPa}$ with a probability of $0.01 \%$.

\section{Conclusion}

Through the research, analysis and calculation of the minimum value distribution of 24 groups of bolts, it can be concluded that:

1) the distribution of the minimum value of bolt tensile strength conforms to Gumbel extreme distribution;

2) when the return period is 10000 , the predicted minimum tensile strength is $1071.7 \mathrm{MPa} \pm 3.2 \mathrm{MPa}, k=2$. 
It is higher than the minimum value of $1040 \mathrm{MPa}$ required by ISO $898-1$ standard and it can be determined that the quality of the bolt is stable and reliable and the specific production process is reliable.

\section{References}

[1] ISO 898-1:2013 Mechanical properties of fasteners made of carbon steel and alloy steel - Part 1: Bolts, screws and studs with specified property classes Coarse thread and fine pitch thread.

[2] Shi Daoji, 2006, Practical statistical method of extreme value. (Tianjin: Tianjin Science and Technology Press). (In Chinese)

[3] Hou Shu-min, LI You-rong, LIU Guang-lin. 2005. Application of K-S Test and Wavelet Analysis in Rolling Bearing Fault Diagnosis. Coal Mine Machinery, 1, 129-130. (In Chinese)

[4] Nan Bo. 2012. Statistical analysis of snow load based on K-S inspection method. Journal of Lanzhou University of technology. 38, 1, 115-119. (In Chinese)

[5] LIU Zhi-fei, YAN Pei-jun, XU Ren-ping, etc. 2013. Effect of Strain Age on Impact Toughness of ADB610 Steel. Development and Application of Materials. 28, 3, 36-39. (In Chinese)

[6] GB 50199-2013 Unified standard for reliability design of hydraulic engineering structures. (In Chinese)

[7] DIAO Hongquan, LIN Jiahui, Yao Huiming. 2019. Statistical parameter estimation method and application of Gumbel distribution. Science and Technology Innovation Herald. 08, 130-132. (In Chinese)

[8] ZHANG Xinsheng, CAO Naining, LI Yayun. 2016. Residual Life Prediction of Buried Oil and Gas Pipelines Based on Gumbel Extreme Value Type I Distribution. Journal of Chinese Society for Corrosion and Protection. 36, 4, 370-374. (In Chinese)

[9] ASTM E2283-08 (2019) Standard Practice for Extreme Value Analysis of Nonmetallic Inclusions in Steel and Other Microstructural Features.

[10] Hong Wei. 1988. The Limited Value Distribution and Application of Annual Lowest Climate Temperature . Journal of Fujian College of Forestry, 8, 3, 229-234. (In Chinese)

[11] IEC/TR 60727-2:1993 Evaluation of electrical endurance of electrical insulation systems - Part 2: Evaluation procedures based on extreme-value distributions.

[12] ISO 3534-1:2006 Statistics - Vocabulary and symbols - Part 1: General statistical terms and terms used in probability.

[13] ISO 5479:1997 Statistical interpretation of data Tests for departure from the normal distribution.

[14] Shi Daoji. 1988. An Analysis of Variance Test for the Extreme Value Distribution. Journal of Tianjin
University, 2, 116-121. (In Chinese) 